\title{
DISTRIBUIÇÃO GEOGRÁFICA POTENCIAL DE MAYTENUS ILICIFLORA (CELASTRACEAE) NO BRASIL FRENTE ÀS MUDANÇAS CLIMÁTICAS
}

\author{
Thays Mayara Camassola ${ }^{(a)}$, Fabiana Gonçalves Barbosa ${ }^{(b)}$ \\ (a) Acadêmica do Curso de Geografia - Bolsista do Programa Institucional de Bolsa de Iniciação Científica \\ (PIBIC) - UNESC - Criciúma, SC, Brasil, camassola.thays@ gmail.com \\ (b) Docente do Programa de Pós-graduação em Ciências Ambientais - UNESC - Criciúma, SC, Brasil, \\ fabibarbos@gmail.com
}

\section{EIXO: BIOGEOGRAFIA, MANEJO DE ÁREAS NATURAIS E PROTEGIDAS: CONSERVAÇÃO DA} BIODIVERSIDADE

\begin{abstract}
Resumo:
As discussões acerca do impacto na biodiversidade mundial causadas pelas alterações climáticas vêm sendo bastante ampliadas. Nos últimos anos, Maytenus ilicifolia Mart. Ex Reissek é uma das espécies mais utilizadas para distintos fins medicinais; sua valorização comercial somada às fronteiras agrícolas disparou o extrativismo, possibilitando reduzir drasticamente a população desta planta. Aliado as futuras alterações climáticas, o risco à conservação aumenta. Neste propósito, utilizamos uma combinação de modelos bioclimáticos para mapear a adequabilidade climática potencial de $M$. ilicifolia no Brasil frente às alterações climáticas. Utilizamos 215 registros de presença de $M$. ilicifolia, quatro diferentes técnicas de modelagem (conduzidas no ambiente R), cinco variáveis bioclimáticas atuais e futuras e três Modelos de Circulação Global na geração dos modelos atuais e futuros. Em conclusão, a adequabilidade climática no futuro (2100) para o Brasil será reduzida nos Estados do Mato Grosso do Sul e Paraná e ampliada em regiões mais frias.
\end{abstract}

Palavras chave: Consenso de predição; Conservação; Modelos bioclimáticos; Planta medicinal.

\section{Introdução}

Conforme alertado por todos os relatórios do IPCC, as alterações climáticas já apresentam indícios em todo o Planeta e seu rápido impacto na biodiversidade será ainda mais agravado no século XXI (VALE; ALVES; LORINI, 2009) pois produzirão alterações mesmo em áreas inferiormente antropizadas (MALCOM et al, 2006). Baseado nestes relatórios, Vale, Alves e Lorini (2009) indicam "que 20 a 30\% das espécies estarão expostas a um alto risco de extinção caso as temperaturas aumentem de 2 a $3^{\circ} \mathrm{C}$ ". Todo este aquecimento modificará não somente o clima, como também a distribuição das espécies (HUGHES, 2000). Estas possuem determinadas respostas de acordo com o nicho ambiental (THUILLER, 2005) ou com características fisiológicas (BEAUMONT; HUGHES, 2002), sendo elas: i) aclimatação, ii) adaptação, iii) dispersão ou iv) extirpação (PETERSON et al. 2001).

Na luta pela preservação, outro agravante para Gaikwad, Wilson e Ranganathan (2011) trata-se da maneira como a informação documentada sobre plantas medicinais é disponibilizada: geralmente de 
XVII Simpósio Brasileiro

de Geografia Fisica Aplicada

I Congresso Nacional

de Geografia Física

\section{OS DESAFIOS DA GEOGRAFIA FÍSICA NA FRONTEIRA DO CONHECIMENTO Instituto de Geociências - Unicamp Campinas - SP \\ 28 de Junho à 02 de Julho de 2017}

forma heterogênea, geograficamente tendenciosa, limitada e fragmentada. Diante desta problemática, "Modelos de nicho ecológico e mapas compostos derivados fornecem um meio útil para a identificação de espécies de plantas medicinais habituais, hotspots e habitats de valor cultural para a priorização de conservação" (GAIKWAD; WILSON; RANGANATHAN, 2011). Tal técnica relaciona registros de ocorrência da espécie com um conjunto de preditores bioclimáticos atuais para encontrar ambientes adequados, onde uma população possa se manter viável (MATEO et al, 2011). Posteriormente, projeta-se no mesmo espaço geográfico sob um cenário de clima futuro para predizer a distribuição potencial futura da espécie (PEARSON; DAWSON, 2003).

No cenário sintetizado pelo Painel Brasileiro de Mudanças Climáticas, baseado no último relatório do IPCC, a biodiversidade Brasileira estará ainda mais vulnerável visto que a pior hipótese estima que o aumento da temperatura do ar até 2100 será entre $1{ }^{\circ} \mathrm{C}$ e $6{ }^{\circ} \mathrm{C}$, em comparação à registrada no fim do século (TOLEDO, 2013). Tal previsão preocupa, pois o país possui uma expressiva fração da diversidade mundial de plantas (FORZZA et al. 2010), inclusive um rico conjunto de espécies medicinais nativas (LORENZI; MATOS, 2008) que são amplamente utilizadas no cotidiano do Brasileiro. Maytenus ilicifolia Mart. ex Reissek (Celastraceae) popularmente conhecida por espinheira-santa, apresenta predileção por clima temperado e subtropical (SANTOS; OLIVEIRA, 2009), ocorrendo, portanto, nos Estados do Mato Grosso do Sul, Paraná, Rio Grande do Sul e Santa Catarina (CARVALHO; OKANO; LEITÃO FILHO, 2004), bem como, nos países vizinhos (leste da Argentina, Paraguai e Uruguai (LOURTEIG; O'DONELL, 1955). Maytenus ilicifolia destaca-se pelo alto valor medicinal no tratamento de problemas do trato gastrointestinal (DI STASI, 2004). Nos últimos anos, M. ilicifolia tem sido uma das espécies de plantas medicinais mais utilizadas em programas de fitoterapia no Brasil (CARVALHO et al, 2008), bem como uma das mais demandadas pelo mercado (STEENBOCK; REIS, 2014), principalmente depois de comprovadas suas propriedades farmacológicas (SANTOS et al, 2009).

Em contraste com a rica diversidade de plantas medicinais, o uso de modelos bioclimáticos no Brasil para este fim - ainda é inexistente. Sua maior aplicação até o momento deu-se no mapeamento da distribuição potencial futura de espécies endêmicas, raras ou ameaçadas de extinção (HOFFMANN et al, 2015), invasoras (LOYOLA et al, 2012), transmissoras de doenças (COSTA et al, 2014) e polinizadoras (GIANNINI et al, 2012). No intuito de garantir a preservação desta espécie perante as alterações climáticas globais, extrativismo e expansão agrícola desordenada, nós utilizamos modelos bioclimáticos para predizer a distribuição geográfica de M.ilicifolia no Brasil frente às alterações climáticas globais.

\section{Material e métodos}


XVII Simpósio Brasileiro

de Geografia Física Aplicada

Congresso Nacional

de Geografia Física

\section{OS DESAFIOS DA GEOGRAFIA FÍSICA NA FRONTEIRA DO CONHECIMENTO \\ Instituto de Geociências - Unicamp \\ Campinas - SP \\ 28 de Junho à 02 de Julho de 2017}

Registros de ocorrência de Maytenus ilicifolia: Montamos um banco de dados com os registros de ocorrência de M. ilicifolia, através de compilação da literatura acadêmica, de exemplares depositados em coleções científicas e de bancos de dados de biodiversidade disponíveis via internet (Global Biodiversity Facility - GBIF (http://www.gbif.org) e SpeciesLink (http://splink.cria.org.br/)).

Preditores bioclimáticos: Nós utilizamos cinco variáveis bioclimáticas atuais e futuras (projetadas para 2100) (média da temperatura anual, variação da temperatura média diurna, isotermalismo, precipitação do trimestre mais chuvoso e precipitação do trimestre mais quente) e três Modelos de Circulação Global (Global Circulation Models - GCMs: CCSM, CNRM, MIROC), ambos pertencentes ao banco de dados EcoClimate.

Modelos bioclimáticos: Para gerar os modelos bioclimáticos atual e futuro (2100) de M. ilicifolia no Brasil nós utilizamos quatro diferentes técnicas de modelagem (BIOCLIM, Distância de Gower, Máquina de Vetores de Suporte (Support Vector Machine: SVM) e Modelo Linear Generalizado). Posteriormente, com o propósito de aumentar a confiabilidade dos resultados gerados pelos modelos escolhidos ou tentar diminuir a incerteza de se utilizar modelos gerados por diferentes algoritmos (GIANINNI et al, 2012), nós combinamos as previsões dos modelos bioclimáticos gerados por cada uma das técnicas de modelagem de forma a produzir um modelo final, ou seja, um mapa consenso da distribuição potencial de $M$. ilicifolia no Brasil frente às mudanças climáticas.

\section{Resultados obtidos}

A partir dos registros de ocorrência de M. ilicifolia (figura 1), nossos resultados apontam para o futuro (2100) no Brasil: (i) uma redução na adequabilidade climática nos Estados do Mato Grosso do Sul e Paraná e (ii) um aumento na adequabilidade climática em regiões mais frias, conforme ilustrado na figura 2: 


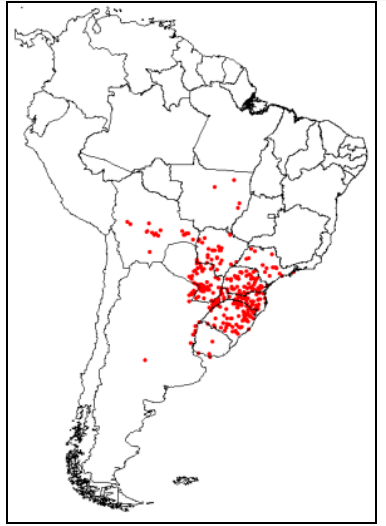

Figura 1 - Registros de ocorrência de $M$. ilicifolia

Fonte: Autores (2016)
28 de Junho à 02 de Julho de 2017

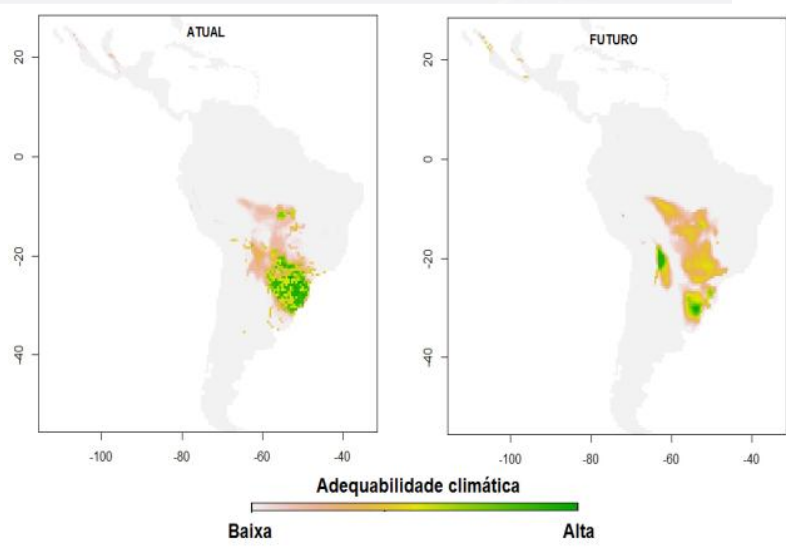

Figura 2 - Modelos bioclimáticos atual e futuro (2100) para Espinheira-santa no Brasil.

Fonte: Autores (2016)

Diante disso, é fácil perceber o imenso desafio que cada país enfrentará para conservar sua biodiversidade não somente com medidas de adaptação, mas de mitigação. (VALE; ALVES; LORINI, 2009). Ademais, pode-se incluir o deslocamento das espécies para um espaço climático mais favorável onde às fronteiras das unidades de conservação não serão respeitadas (LEMES; LOYOLA, 2014).

Nosso estudo destaca a capacidade de uma combinação de modelos bioclimáticos como uma ferramenta útil e rentável para mapear a adequabilidade climática potencial de Maytenus ilicifolia no Brasil e, consequentemente, nossos resultados podem subsidiar uma proposta de manejo mais eficaz, aumentando a probabilidade de persistência desta espécie no futuro.

\section{Fonte financiadora: PIBIC/ UNESC}

\section{Bibliografia}

BEAUMONT, L. J; HUGHES, L. Potential changes in the distributions of latitudinal restricted Australian butterfly species in response to climate change. 2002. Global Change Biology, 8:954-971.

CARVAlHO, A. C. B; BALBINO, E.E; MACIEL, A; PERFEITO, J. P. S. (2008) Situação do registro de medicamentos fitoterápicos no Brasil. Revista Brasileira Farmacognosia, 18:314- 319.

CARVAlHO-OKANO, R. M; LeITÃo FILHO, H. F. (2004) O gênero Maytenus Mol. emend. Mol. (Celastraceae) no Brasil extra-amazônico. In: Reis MS \& Silva SR (Organizadores). Conservação e uso sustentável de plantas medicinais e aromáticas: Maytenus spp., espinheira-santa. Brasília: IBAMA. 11-51p.

COSTA. J; DORNAK, L. L; ALMEIDA, C. E, PETERSON AT. (2014) Distributional potential of the Triatoma brasiliensis species complexa at presente and under scenarios of future climate conditions. Parasites \& Vectors, $7: 238$. 
DI STASI, L. C (2004) Aspectos químicos e farmacológicos da espinheira-santa: uma análise da utilidade dos dados. In: Reis MS \& Silva SR (Organizadores). Conservação e uso sustentável de plantas medicinais e aromáticas: Maytenus spp., espinheira santa. Brasília: IBAMA. 67-92p.

FORZZA, R. C (2010) Catálogo de plantas e fungos do Brasil. Rio de Janeiro: Instituto de Pesquisa Jardim Botânico do Rio de Janeiro. 871p

GAIKWAD, J; WILSON, P. D; RANGANATHAN, S. Ecological niche modeling of customary medicinal plant species used by Australian Aborigines to identify species-rich and culturally valuable areas for conservation. Ecological Modelling, 222: 3437-3443.

GIANNINI, T. C et (2012). Pollination services at risk: bee habitats will decrease owing to climate change in

Brazil. Ecological Modelling 244: 127-131.

HUGHES, L. (2000) Biological consequences of global warming: is the signal already apparent. Trends in Ecology and Evolution, 15:56-61.

LEMES, P; LOYOLA, R; Mudanças climáticas e prioridades para conservação da biodiversidade. Revista de Biologia Neotropical, Goiânia, p.47-57, 18 jul. 2014. Semestral. Disponível em: <https://www.revistas.ufg.br/RBN>. Acesso em: 19 dez. 2016.

LOURTEIG, A; O’DONNELL, C. A (1955) Las Celastraceas de Argentina y Chile. Natura, 1:181-233.

LOYOLA, R. D et al (2012) Climate change might drive species into reserves: a case study of the American bullfrog in the Atlantic Forest Biodiversity Hotspot. Alytes, 29:61-74.

MALCOM, J.R; LIU, C.;NELSON, R.P.; HANSEN, L. \& HANNAH, L. 2006. Global Warming and Extictions os Endemic Species from Biodiversity Hotspots. Conservation Biology, 20(2): 538-548. Mateo RG, Felicíssimo AM \& Munhoz J. (2011) Modelos de distribución de especies: una revision sintética. Revista Chilena de Historia Natural, 84: 217-240.

MATOS, F. J. A.et al. Plantas medicinais no brasil: nativas e exóticas. 2. ed. Sao Paulo: Harri Lorenzi, 2008.

PEARSON, R; DAWSON, T (2003) Predicting the impacts of climate change on the distribution of species: are cioclimatic envelope models useful. Global Ecology and Biogeography, 12: 361-371.

PETERSON, A. T (2001) Effects of global climate change on geographic distributions of Mexican Cracidae. Ecological Modelling, 144: 21-30.

R CORE TEAM (2014) R: a language and environment for statistical computing. R Foundation for Statistical Computing, Vienna, Austria. URL http://www.R-project.org/.

SANTOS OLIVEIRA, R; COULAUD-CUNHA, S; COLAÇO, W. (2009) Revisão da Maytenus ilicifolia Mart. Ex Reissek, Celastraceae. Contribuição ao estudo das propriedades farmacológicas. Revista Brasileira de Farmacognosia, 19: 650-659. 
STEENBOCK, W; REIS, M.S (2004) Manejo sustentável de populações naturais de espinheira-santa. In Reis MS \& Silva SR (Organizadores). Conservação e uso sustentável de plantas medicinais e aromáticas: Maytenus spp., espinheira-santa. Brasília: IBAMA. 145-161p.

THUILLER, W. et al. (2005) Niche-based modeling as tool for predicting the risk of alien plant invasions at a global scale. Global Change Biology, vol. 11, p. 2234-2250.

TOLEDO, K. Quinto relatório do IPCC mostra intensificação das mudanças climáticas. 2013. Disponível em: http://agencia.fapesp.br/quinto_relatorio_do_ipcc_mostra_intensificacao_das_mudancas_climaticas/17944/>. Acesso em: 10 out. 2016.

VALE, M.M; ALVES, M.A.S; LORINI, M.L. 2009. Mudanças climáticas: desafios e oportunidades para a conservação da biodiversidade brasileira. Oecol. Bras. 13(3):518-53. 